This item was submitted to Loughborough's Research Repository by the author.

Items in Figshare are protected by copyright, with all rights reserved, unless otherwise indicated.

\title{
Phased mission modelling using fault tree analysis
}

PLEASE CITE THE PUBLISHED VERSION

PUBLISHER

() IMechE / Professional Engineering Publishing

LICENCE

CC BY-NC-ND 4.0

REPOSITORY RECORD

La Band, Rachel A., and J.D. Andrews. 2008. "Phased Mission Modelling Using Fault Tree Analysis". figshare. https://hdl.handle.net/2134/3938. 
This item was submitted to Loughborough's Institutional Repository (https://dspace.lboro.ac.uk/) by the author and is made available under the following Creative Commons Licence conditions.

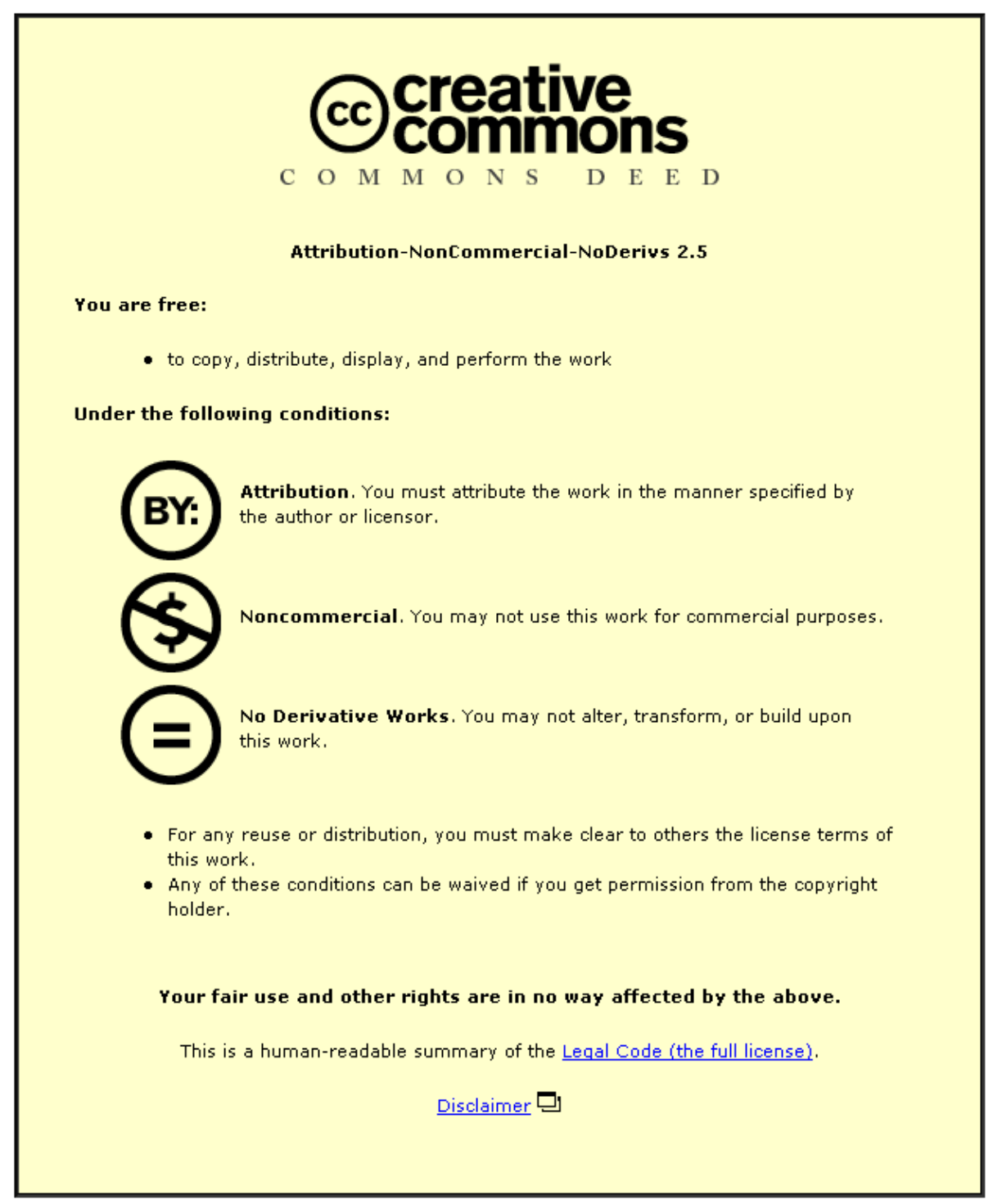

For the full text of this licence, please go to: http://creativecommons.org/licenses/by-nc-nd/2.5/ 


\title{
Phased mission modelling using fault tree analysis
}

\author{
R A La Band and J D Andrews* \\ Department of Aeronautical and Automotive Engineering, Loughborough University, Leicestershire, UK
}

\begin{abstract}
Many types of system operate for missions that are made up of several phases. For the complete mission to be a success, the system must operate successfully during each of the phases. Examples of such systems include an aircraft flight, and also many military operations for both aircraft and ships. An aircraft mission could be considered as the following phases: taxiing to the runway, take-off, climbing to the correct altitude, cruising, descending, landing and taxiing back to the terminal. Component failures can occur at any point during the mission, but their condition may only be critical for one particular phase. As such, it may be that the transition from one phase to another is the critical event leading to mission failure, and the component failures resulting in the system failure may have occurred during some previous phase. This paper describes a means of analysing the reliability of non-repairable systems that undergo phased missions. Fault tree analysis (FTA) has been used as a method for assessing the system performance. The results of the analysis are the system failure modes in each phase (minimal cut sets), the failure probability in each phase and the total mission unreliability. To increase the efficiency of the analysis, the fault trees constructed to represent the system failure logic are analysed using a modularization method. Binary decision diagrams (BDDs) are then employed to quantify the likelihood of failure in each phase.
\end{abstract}

Keywords: phased missions, fault tree analysis, binary decision diagrams

\section{INTRODUCTION}

If the success of a mission is reliant upon a sequential set of objectives operating over different time intervals, it may be referred to as a phased mission. During the execution of the phases in a mission, the system is altered such that the logic model, system configuration or system failure characteristics may change to accomplish a different objective. The phases in a mission may be expressed in terms of phase number, time interval, system configuration, task(s) to be undertaken, performance measure(s) of interest and maintenance policy. This type of mission can be characterized as a sequence of discrete events required to complete a task, e.g. an aircraft flight phase pattern.

In order to identify possible causes of phase and mission failure, a method is required to express how combinations of component failures (basic events) can occur during the phases throughout the mission and cause system failure.

The MS was received on 28 April 2003 and was accepted after revision for publication on 24 February 2004.

*Corresponding author: Department of Aeronautical and Automotive Engineering, Loughborough University, Loughborough, Leicestershire LE11 3TU, UK.
These failure events then require quantification to enable the likelihood and frequency of mission failure to be determined.

The main techniques that have previously been implemented for the solution to phased mission problems are that of fault tree analysis (FTA), Markov analysis and simulation. The technique of fault tree analysis is a commonly used tool to assess the probability of failure of industrial systems. This method may be adapted for analysis of systems comprising more than one phase, where each phase depends on a different logic model. Hence, the complexity of the modelling is significantly more difficult than for single-phase systems. The fault tree approach represents the failure logic of the system in an inverted tree structure, and allows for both qualitative and quantitative system reliability analysis to take place. The earliest inspection of the analysis of phased missions was that carried out by Esary and Ziehms [1]. This research employed a fault tree method by which the mission is split into consecutive phases whereby each phase performs a specified task. The success of the mission depends on the performance of the non-repairable components used in each phase. The probability of this success is referred to as the mission reliability. Mission unreliability is defined as the probability that the system fails to function successfully during at least one phase of the mission. 
An important problem is to calculate, as efficiently as possible, either the exact value or bounds for the mission unreliability parameter. Methods to obtain estimates of such bounds are discussed by Burdick et al. [2].

Situations may be encountered in phased mission analysis that prevent the assumption of independence between component failure or repair being made. In such circumstances, methods other than fault tree analysis must be applied. One such technique is the Markov approach [3]. The reliability of a mission may not be obtained by the simple multiplication of the individual phase reliabilities. This is due to the fact that, at the phase change times, the system must occupy a state that allows both of the involved phases to function. The phases of the mission will be statistically dependent, and an approach for solution has been presented by Smotherman and Zemoudeh [4] for repairable components. Of the many considered solutions to phased mission problems, simulation techniques typically offer the greatest generality in representation but are also often the most expensive in computational requirements. The Markov method offers a combination of flexibility in representation and ease of solution but requires transition rates to be independent of time [5], and suffers from a potential explosion in the number of state equations for even moderately sized problems.

In some situations, it will be difficult to model a system by fault tree or Markov methods. This type of situation will occur if a system is too complex to use deterministic analysis, or if the failure and repair distributions of a component may not have a constant failure or repair rate. In such circumstances, simulation may be necessary.

Previous work has concentrated on assessing mission success. This paper identifies the probability of failure in each phase. Depending on the phase at which the failure occurs, the consequences can be significantly different. Having calculated the probability of failure in each phase, the mission unreliability is simply the sum of the phase failure probabilities. In reducing the complexity of the problem in this way, the efficiency of the approach is improved. Further improvement can be achieved by employing modularization methods and the binary decision diagram (BDD) method. Focus will be restricted to a system where components are non-repairable.

\section{PREVIOUS FAULT TREE METHODS FOR PHASED MISSIONS}

A very simple phased mission problem consisting of non-repairable components with $A, B$ and $C$ representing component failures in each of the phases may be used to demonstrate approaches to phased mission analysis (Fig. 1). The simple system will enable the features of the approaches to be understood without complicated analysis. During phase 1 , which lasts until time $t_{1}$, the success of the mission is dependent upon the success of all of the three components $A, B$ and $C$. Successful

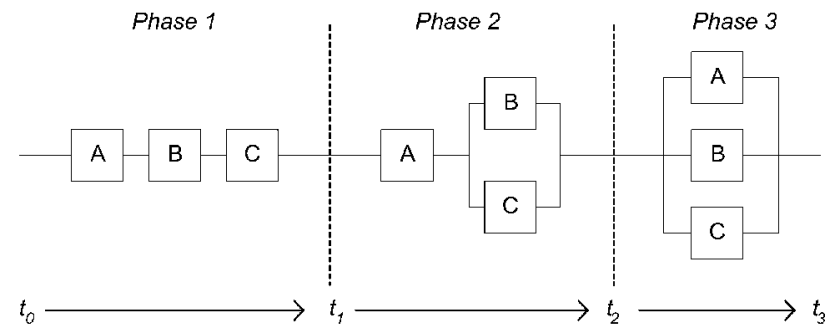

Fig. 1. Reliability network of a simple phased mission system

completion of phase 1 means the system then enters phase 2 , which requires component $A$ to function between times $t_{1}$ and $t_{2}$, along with at least one of the remaining two components $B$ and $C$. The final phase requires only one out of the three components to function between $t_{2}$ and $t_{3}$ for the mission to be accomplished successfully.

Considering the phases as separate systems, the fault trees to represent individual phase failure are as shown in Fig. 2. The notation used to represent component failure in phase $i$ is $A_{i}, B_{i}$, and $C_{i}$ for components $A, B$ and $C$ respectively.

The minimal cut sets for each phase when treated as separate systems are as follow:

\begin{tabular}{lll}
\hline Phase 1 & Phase 2 & Phase 3 \\
\hline$A$ & $A$ & $A B C$ \\
$B$ & $B C$ & \\
$C$ & & \\
\hline
\end{tabular}

The method for calculating the reliability of a phased mission cannot simply be obtained by the multiplication of the reliabilities of each of the individual phases as this involves the false assumptions that the phases are independent and all components are in the working state at the beginning of each phase, and results in an appreciable overprediction of system reliability.

A method proposed by Esary and Ziehms [1] involves the transformation of a multiphase mission to that of an equivalent single-phase mission. This transformation process
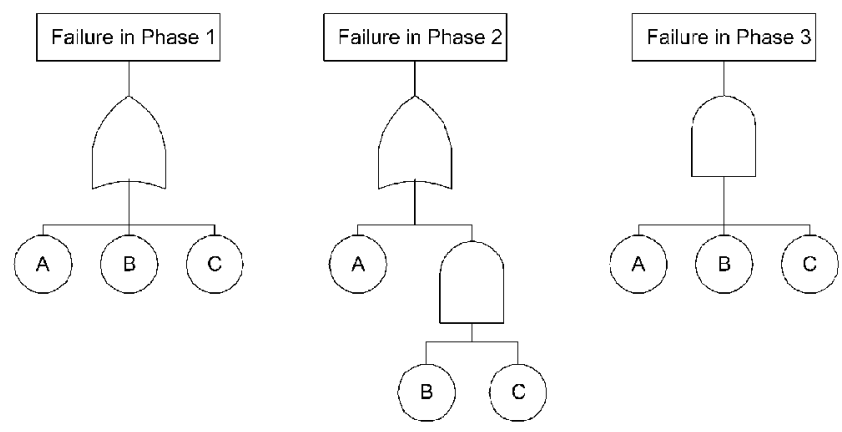

Fig. 2. Fault tree representation of individual phase failures 
involves three stages and is only concerned with the failure of the mission. It does not account for the phase in which failure occurs. Having expressed the failure causes for each phase by separate fault trees as in Fig. 2, the transformation to single phased mission is achieved by:

1. Elimination of unnecessary cut sets. If cut sets of an earlier phase contain any from a later phase, they may be removed from the first. For example, if the minimal cut sets for each phase in the mission are:

\begin{tabular}{ll}
\hline Phase 1 & Phase 2 \\
\hline$A B$ & $A$ \\
$\ldots$ & $\ldots$ \\
$\ldots$ & $\ldots$ \\
$C D E$ & $C F$ \\
\hline
\end{tabular}

then minimal cut set $A B$ can be removed from phase 1 as $A$ failing in phase 1 means it will still be failed in phase 2 which will cause the mission to fail. This makes the status of component $B$ irrelevant. In the problem shown in Fig. 2, it means that minimal cut set $A$ can be removed from phase 1 .

2. Component failure events in each phase fault tree are replaced by an OR combination of the failure events for that and all preceding phases. For example, component $A$ failure in phase 2 would be represented by the OR of the failure of the component in phase $1, A_{1}$, and in phase $2, A_{2}$, since the component is non-repairable (see Fig. 3). [Note that the replacement is only performed on phase fault trees that have the eliminated minimal cut sets removed.]

3. Each phase failure is combined using an OR gate to represent overall mission failure (i.e. the event that any phase does not complete successfully). This transforms the original multiphase mission into an equivalent single-

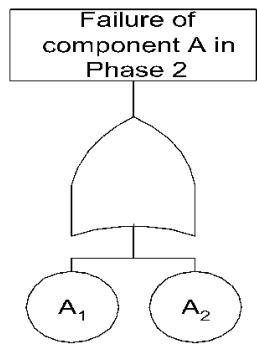

Fig. 3. Replacement OR combination

phase mission as shown in Fig. 4. This equivalent singlephase mission (see Fig. 4) produces different minimal cut sets than would have resulted from the combination of the individual phase minimal cut sets.

The process of removing cut sets prior to the construction of fault trees can generally be seen to reduce the complexity of the problem for analysis. However, since cuts sets are removed to produce a single-phase mission, it becomes impossible to calculate individual phase failure probabilities. Since failures in the different phases may have different consequences, it is advantageous if the probability of failure in any phase can be calculated.

\section{PROPOSED FAULT TREE METHOD FOR PHASED MISSIONS}

A new method is proposed that enhances the fault tree approach in the previous section. It will enable the probability of failure in each phase to be determined in addition to the whole mission unreliability. For any phase, the method combines the causes of success of previous phases

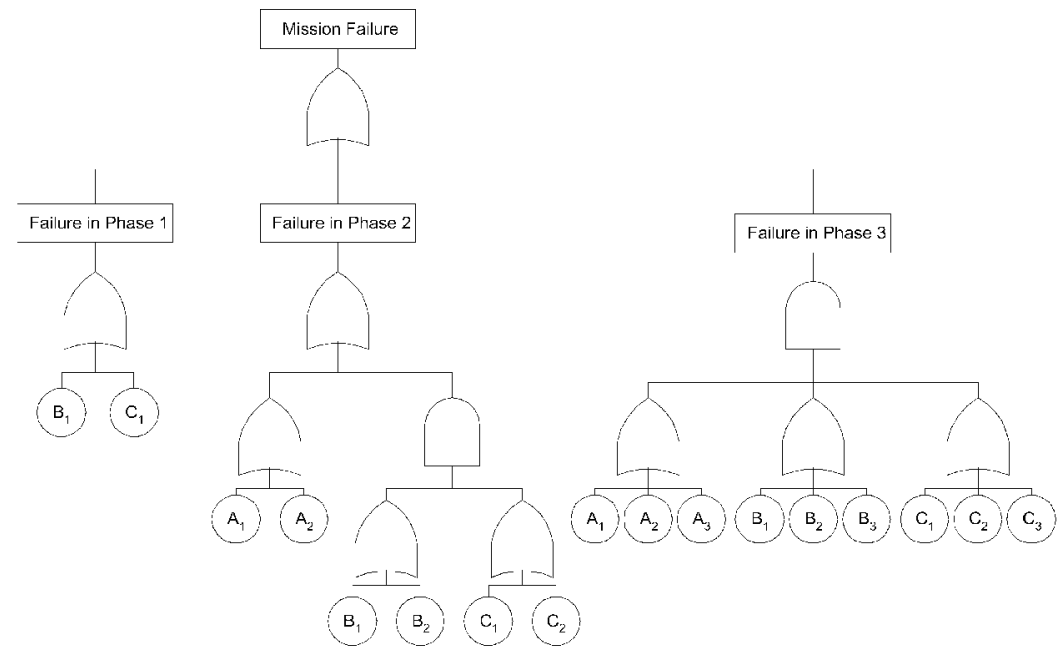

Fig. 4. Equivalent single-phase mission 
with the causes of failure for the phase being considered to allow both qualitative and quantitative analysis of both phase failure and mission failure.

The event of component failure in phase $i$ is again represented as the event that the component could have failed during any phase up to and including phase $i$. System failure in phase $i$ is represented by the AND of the success of phases 1 to $i-1$ and the failure during phase $i$. (Fig. 5). (Note that the symbol at the output of the lower events, failure in phase 1 to $i-1$, on the left-hand branch is a NOT gate.)

Mission unreliability, $Q_{\text {MIss }}$, is then obtained from

$$
Q_{\mathrm{MISS}}=\sum_{i=1}^{n} Q_{i}
$$

where $Q_{i}$ is the failure probability in phase $i$ and $n$ is the total number of phases.

This method allows for the evaluation of individual phase failures, and also accounts for the condition where components are known to have functioned to enable the system to function in previous phases. However, owing to the fact that cut sets are not removed until a later stage in the analysis, the fault tree can be much more complex and require significantly more effort to solve.

The failure of a system may occur in many different ways. Each unique way is referred to as a system failure mode and involves either the failure of a single component or a combination of failures of multiple components. To determine the minimal cut sets of a phase or mission, either a top-down or a bottom-up approach is applied to the relevant fault tree. For any phases after the first phase, the incorporation of the success of previous phases means that the fault tree will be non-coherent and not simply consist of AND and OR gates.
NOT logic will be required to represent this success, and the combinations of basic events that lead to the occurrence of the top event are referred to as prime implicants.

This proposed method may be applied for the simple three-phase mission given in Fig. 1. The fault tree to represent the initial phase failure of the mission remains identical to the fault tree representation of the individual phase failure of phase 1 shown in Fig. 2. Phase 2 failure can then be shown as the combination of phase 1 success and failure in phase 2 (Fig. 6). Similarly, phase 3 failure can be represented as the combination of phase 1 and phase 2 successes, and failure in phase 3 (Fig. 7).

\subsection{Fault tree modularization}

Fault tree modularization techniques are helpful to reduce the size of a fault tree to enable prime implicants to be found more efficiently. These modularization techniques reduce both memory and time requirements. A non-coherent extension of a modularization technique has been employed in this work [6]. It repeatedly applies the stages of contraction, factorization and extraction to reduce the complexity of the fault tree diagram. The phases are identified as:

1. Contraction. Subsequent gates of the same type are contracted to form a single gate. The resulting tree structure is then an alternating sequence of OR and AND gates.

2. Factorization. Identification of basic events that always occur together in the same gate type. The combination of events and gate type is replaced by a complex event. However, since NOT logic is included in order to combine phase success and failure, in this stage the primary basic events that are found always to occur together in one gate

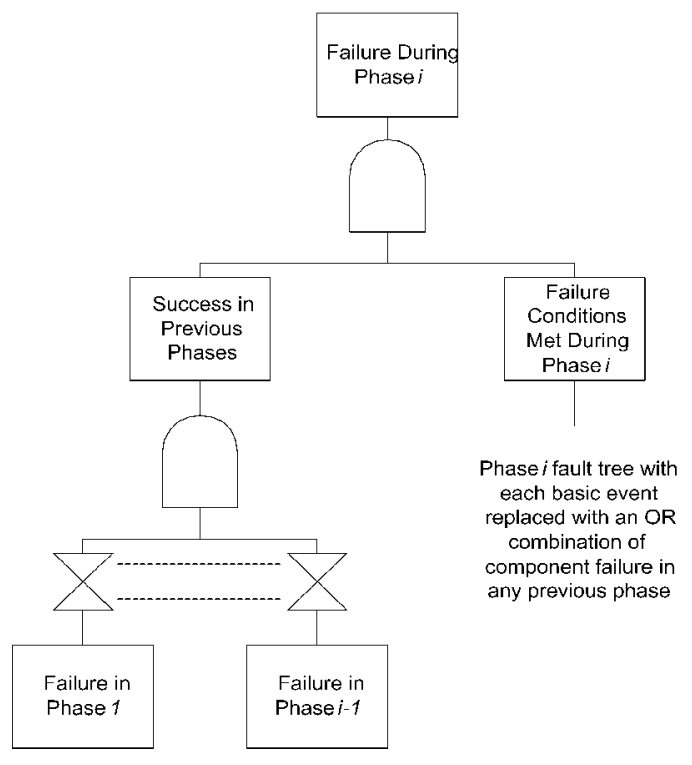

Fig. 5. Generalised phase failure fault tree

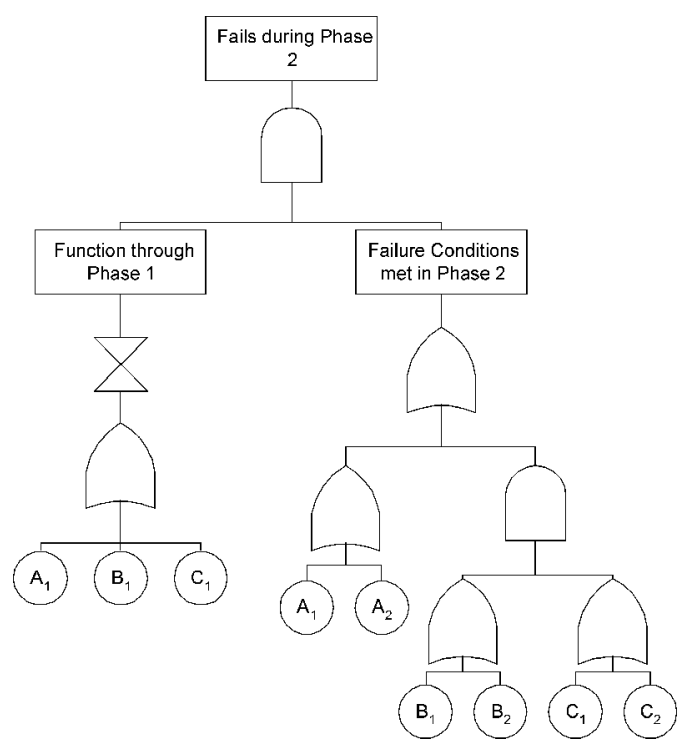

Fig. 6. Phase 2 failure fault tree 

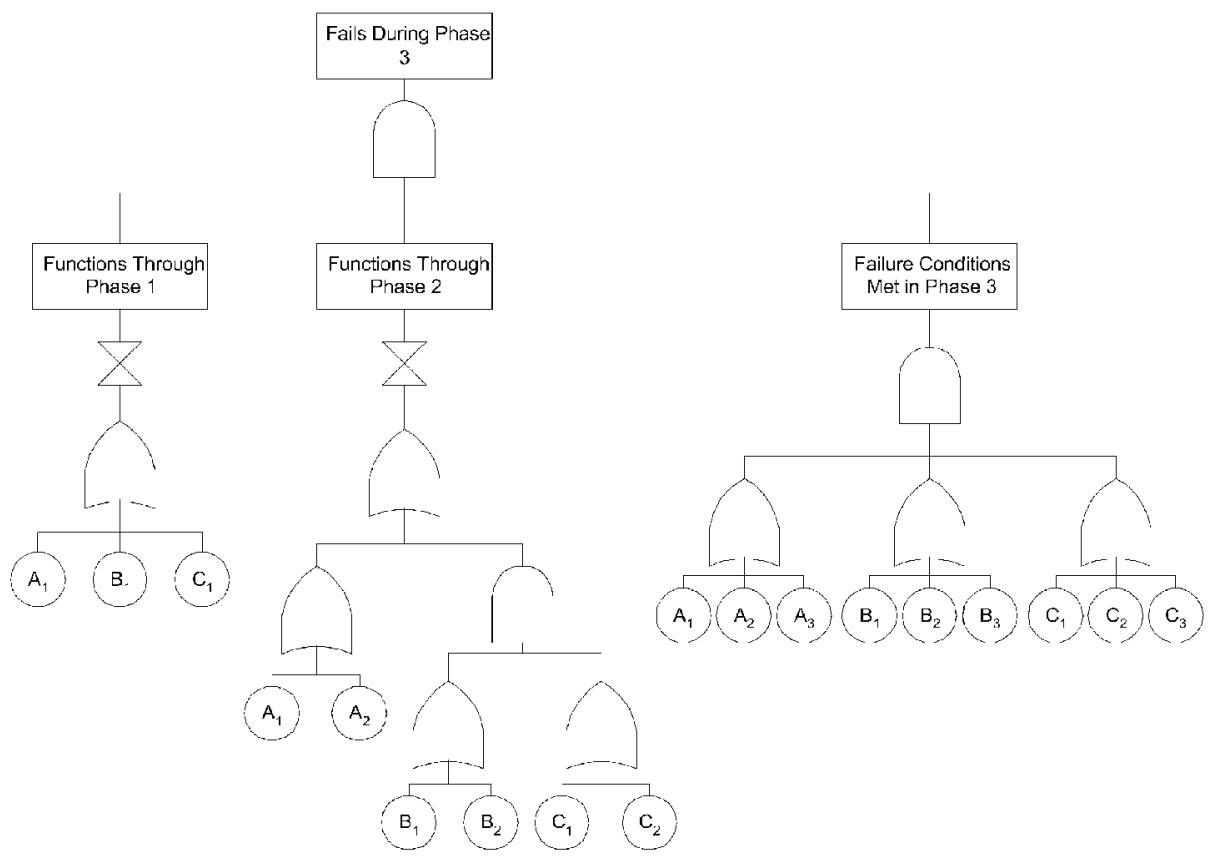

Fig. 7. Phase 3 failure fault tree

type must have complements that always occur together in the opposite gate type by De Morgans' laws, e.g.

$$
\begin{array}{ll}
2000=A+B, & 2001=A \cdot B \\
\overline{2000}=\bar{A} \cdot \bar{B}, & \overline{2001}=\bar{A}+\bar{B}
\end{array}
$$

3. Extraction. Searches for structures within the tree of the form shown in Fig. 8 that may be simplified by extracting an event to a higher level.

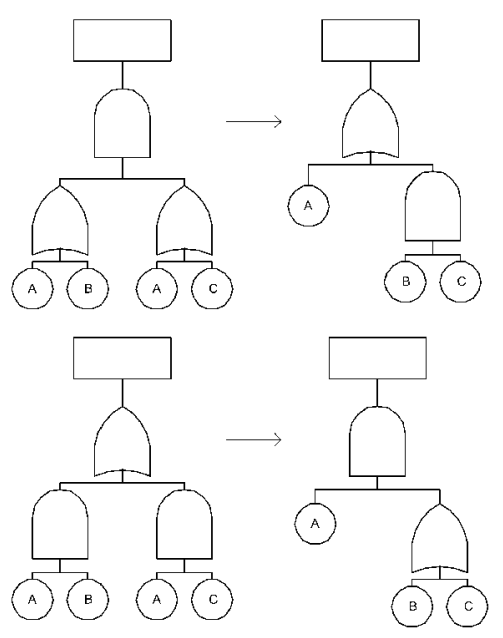

Fig. 8. Extraction stage of the modularization technique

\subsection{Prime implicants in phased mission systems}

Owing to the non-coherent nature of the fault trees, the combinations of basic events that lead to the occurrence of the top event of any phase failure are expressed as prime implicants. The notation used to represent the failure of component $A$ in phase $i$ is $A_{i} ; \bar{A}_{i}$ represents the functioning of component $A$ throughout phase $i$. The notation used to indicate the failure of a component in phase $i$ to $j$ is $A_{i j}$, i.e. the component fails at some time from the start of phase $i$ to the end of phase $j$.

This notation makes it possible to define a new algebra over the phases to manipulate the logic equations. What is of concern in later phases is the time duration (i.e. phases) during which the component failures occur. Therefore, if a combination of events for component $A$ is produced

$$
\overline{A_{1}} \overline{A_{2}}\left(A_{3}+A_{4}\right)
$$

the top event being developed will only be produced if $A$ fails in phases 3 or 4 , i.e. $A_{34}$, where

$$
q_{A_{34}}=q_{A}\left(t_{2}, t_{4}\right)=\int_{t_{2}}^{t_{4}} f_{A}(t) \mathrm{d} t
$$

and $f_{A}(t)$ is the density function of failure times for component $A$. 
The new algebraic laws can be summarized as follows:

$$
\begin{aligned}
& A_{i} \cdot A_{i}=A_{i} \\
& A_{i} \cdot A_{j}=0 \\
& A_{i} \cdot A_{i j}=A_{i} \\
& \overline{A_{i}} \cdot A_{i}=0 \\
& \overline{A_{i}} \cdot A_{i j}=A_{i+1, j} \\
& \overline{A_{i}} \overline{A_{i+1}} \ldots \overline{A_{j}}=\overline{A_{i j}} \\
& A_{i}+A_{i+1}+\cdots+A_{j}=A_{i j}
\end{aligned}
$$

Therefore, if two implicant sets contain exactly the same components where all but one occur over the same time intervals and the other is a failure in contiguous phases, the two implicant sets may be combined with the period of failure for the component with time discrepancy adjusted, e.g.

$$
\begin{aligned}
& A_{1} B_{1} \\
& A_{1} B_{2}
\end{aligned} \rightarrow A_{1} B_{12}
$$

As the components are non-repairable, the event of component failure will only be possible over contiguous phases. This simplification approach therefore allows the prime implicants for the simple example given in Fig. 1 to be expressed as follows:

Phase 1

$$
T_{1}=A_{1}+B_{1}+C_{1}
$$

$\begin{array}{ll} & A_{1} \\ \text { Minimal cut sets: } & B_{1} \\ & C_{1}\end{array}$

Phase 2

$$
\begin{aligned}
& \begin{aligned}
T_{2} & =\overline{A_{1}} \overline{B_{1}} \overline{C_{1}}\left(A_{1}+A_{2}+B_{1} C_{1}+B_{1} C_{2}+B_{2} C_{1}+B_{2} C_{2}\right) \\
& =\overline{A_{1}} A_{2} \overline{B_{1}} \overline{C_{1}}+\overline{A_{1}} \overline{B_{1}} \overline{C_{1}} B_{2} C_{2} \\
& =A_{2} \overline{B_{1}} \overline{C_{1}}+\overline{A_{1}} B_{2} C_{2}
\end{aligned} \\
& \text { Prime implicants: } \quad \overline{A_{2}} \overline{B_{1}} \overline{C_{1}} \\
& \overline{A_{1}} B_{2} C_{2}
\end{aligned}
$$

Phase 3

$$
\begin{aligned}
& T_{3}=\overline{A_{1}} \overline{B_{1}} \overline{C_{1}}\left(\overline{A_{1}} \overline{A_{2}}\left(\overline{B_{1}} \overline{B_{2}}+\overline{C_{1}} \overline{C_{2}}\right) \cdot\left(A_{1}+A_{2}+A_{3}\right)\right. \\
& \left.\cdot\left(B_{1}+B_{2}+B_{3}\right) \cdot\left(C_{1}+C_{2}+C_{3}\right)\right) \\
& =A_{3} B_{3} C_{23}+A_{3} B_{23} C_{3} \\
& \text { Prime implicants: } \begin{array}{llr}
A_{3} B_{3} C_{23} & \begin{array}{l}
A_{3} B_{3} C_{2} \\
A_{3} B_{3} C_{3}
\end{array} \begin{array}{r}
A_{3} B_{3} C_{2} \\
A_{3} B_{2} C_{3} \\
A_{3} B_{3} C_{3}
\end{array} A_{3} C_{3} B_{2} C_{3}
\end{array}
\end{aligned}
$$

\subsection{Quantification}

Having established the prime implicants for each phase, they may now be used to quantify the probability of phase and mission failure. The unreliability, $Q_{i}$, for each individual phase $i$ is found using a simple inclusion-exclusion expansion for the prime implicants $C_{j}$ in the phase

$$
\begin{aligned}
Q_{i}= & \sum_{j=1}^{N_{i}} P\left(C_{j}\right)-\sum_{j=1}^{N_{i}} \sum_{k=1}^{j-1_{i}} P\left(C_{j} \cap C_{k}\right)+\cdots+(-1)^{N_{i}-1} P \\
& \times\left(C_{1} \cap C_{2} \cap \cdots \cap C_{N_{i}}\right)
\end{aligned}
$$

Therefore, the event of phase failure for this simple threephase mission may be expressed as

Phase 1

$$
\begin{aligned}
Q_{1}= & q_{A_{1}}+q_{B_{1}}+q_{C_{1}}-q_{A_{1}} q_{B_{1}}-q_{A_{1}} q_{C_{1}}-q_{B_{1}} q_{C_{1}} \\
& +q_{A_{1}} q_{B_{1}} q_{C_{1}}
\end{aligned}
$$

Phase 2

$$
Q_{2}=q_{A_{2}}\left(1-q_{B_{1}}\right)\left(1-q_{C_{1}}\right)+\left(1-q_{A_{1}}\right) q_{B_{2}} q_{C_{2}}-q_{A_{2}} q_{B_{2}} q_{C_{2}}
$$

Phase 3

$$
Q_{3}=q_{A_{3}} q_{B_{3}} q_{C_{23}}+q_{A_{3}} q_{B_{23}} q_{C_{3}}-q_{A_{3}} q_{B_{3}} q_{C_{3}}
$$

As the failure of each of the phases produces mutually exclusive causes, the probability of mission failure may be expressed as the sum of the unreliabilities of the individual phases

$$
Q_{\mathrm{MISS}}=\sum_{i=1}^{n} Q_{i}
$$

For systems with non-repairable components, the expected number of failures per mission is equal to the mission unreliability.

\section{BINARY DECISION DIAGRAM ANALYSIS FOR PHASED MISSIONS}

A fault tree structure very efficiently represents system failure logic but is not an ideal form for mathematical analysis. Binary decision diagrams represent a logic expression and offer efficient mathematical manipulation, although it is very difficult to construct directly from the system definition. For larger fault trees it is more efficient to convert to a BDD prior to analysis. The approach of performing the quantification process after first converting the fault tree to a BDD form offers significant advantages for large complex fault trees. This is particularly true of structures that are non-coherent, such as the phase failure fault trees.

Figure 9 shows a binary decision diagram. Paths through the BDD start at the top root node and terminate at one of two terminal nodes, 1 or 0 . A terminal 1 indicates top 


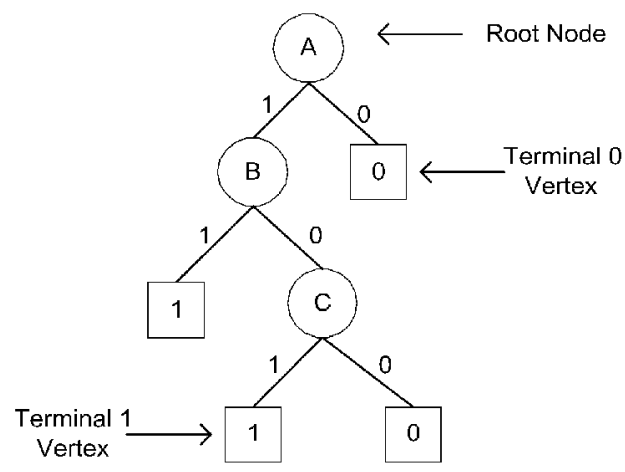

Fig. 9. Binary decision diagram

event occurrence and a terminal 0 indicates top event nonoccurrence.

Each node on the diagram corresponds to a basic event in the fault tree that has to be placed in an ordering prior to BDD construction. In this case the ordering is $A<B<C$. All nodes have two suit branches, a 1 branch corresponds to component failure and a 0 branch corresponds to a component working state.

Prime implicants are given by events on paths through the diagram that lead to a terminal 1 vertex, i.e.

$A B$

In this case, by consensus these can be reduced to minimal cut sets

$$
\begin{aligned}
& A B \\
& A C
\end{aligned}
$$

since this BDD does not represent a non-coherent system. More details of qualitative BDD analysis can be found in reference [7].

\subsection{Construction}

A binary decision diagram consists of vertices where each vertex has an if-then-else structure as shown in Fig. 10.

This if-then-else structure is represented in shorthand (ite) notation as

$$
\operatorname{ite}(X 1, f 1, f 2)
$$

To combine two basic events using a logical operation $\oplus$ (where $\oplus$ represents logical operator AND or OR)

$$
\begin{aligned}
& \text { If } J=\operatorname{ite}(X, f 1, f 2) \\
& \text { and } H=\operatorname{ite}(Y, g 1, g 2) \\
& \text { If } X<Y, \quad J \oplus H=\operatorname{ite}(X, f 1 \oplus H, f 2 \oplus H) \\
& \text { If } X=Y, \quad J \oplus H=\operatorname{ite}(X, f 1 \oplus g 1, f 2 \oplus g 2)
\end{aligned}
$$

The basic events in any BDD are represented as $A=$ ite $(A, 1,0)$ and $\bar{A}=\mathrm{ite}(A, 0,1)$. For phased mission systems, the BDD is constructed so that each component is considered in each phase (in phase order) before the next basic event is taken into account.

\subsection{Quantification}

Since each path to a terminal 1 is disjoint, the top event probability $Q$ is given by

$$
Q_{\mathrm{EXACT}}=\sum_{i=1}^{n} p\left(r_{i}\right)
$$

where $p\left(r_{i}\right)$ is the probability of the $i$ th disjoint path to a terminal 1. Further details of BDD quantification can be found in reference [8].

\subsection{Example}

The simple three-phase mission illustrated in Fig. 1 may be represented in BDD form for each phase and then quantified. The fault trees for phase 1 (Fig. 2), phase 2 (Fig. 6) and phase 3 (Fig. 7) are first converted to BDDs. These BDDs are shown in Figs. 11 to 13 respectively. Their analyses are presented below.

In BDD methodology, to evaluate the success of a phase as opposed to the failure, a 1 is replaced by a 0 , and a 0 by a 1 for the terminal nodes:

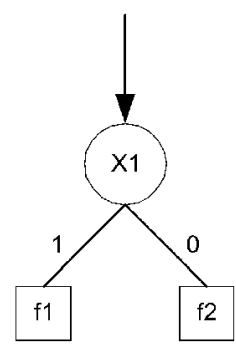

Fig. 10. Binary decision diagram vertex

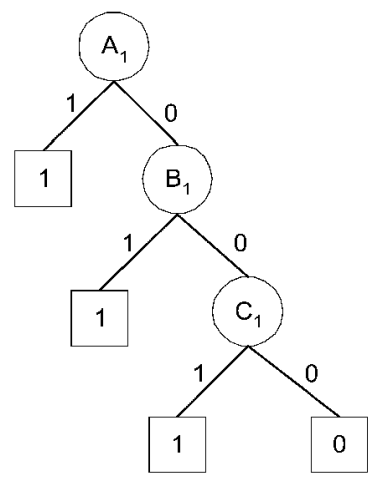

Fig. 11. Phase 1 failure BDD 


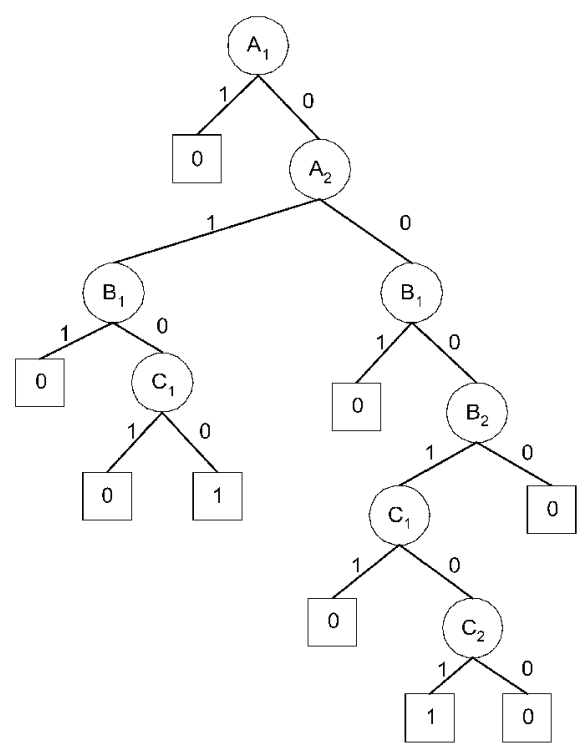

Fig. 12. Failure during phase $2 \mathrm{BDD}$

Phase 1

For phase 1, the ite structure represented by the BDD in Fig. 11 is

ite $\left(A_{1}, 1, \operatorname{ite}\left(B_{1}, 1, \operatorname{ite}\left(C_{1}, 1,0\right)\right)\right)$

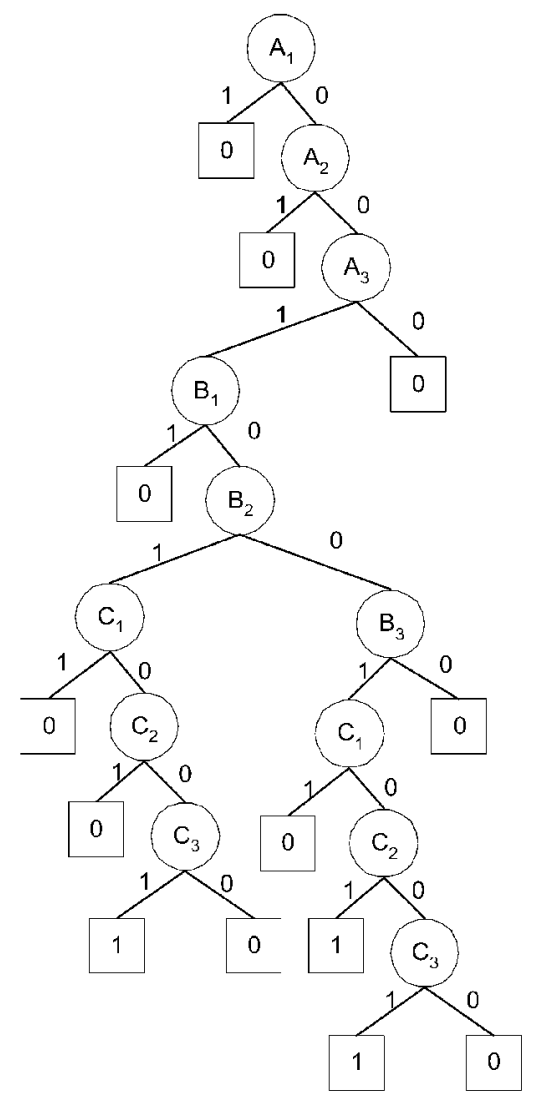

Fig. 13. Failure during Phase 3 BDD
Analysis of this BDD gives

$$
\begin{gathered}
\text { Minimal cut sets: } \quad \begin{array}{l}
A_{1} \\
B_{1} \\
C_{1} \\
Q_{1}=q_{A_{1}}+\left(1-q_{A_{1}}\right) q_{B_{1}}+\left(1-q_{A_{1}}\right)\left(1-q_{B_{1}}\right) q_{C_{1}}
\end{array}
\end{gathered}
$$

Phase 2

The BDD for failure during phase 2 is given by the following ite structure

$$
\begin{aligned}
& \operatorname{ite}\left(A_{1}, 0, \operatorname{ite}\left(A_{2}, \operatorname{ite}\left(B_{1}, 0, \operatorname{ite}\left(C_{1}, 0,1\right)\right),\right.\right. \\
& \left.\left.\operatorname{ite}\left(B_{1}, 0, \operatorname{ite}\left(B_{2}, \operatorname{ite}\left(C_{1}, 0, \operatorname{ite}\left(C_{2}, 1,0\right)\right), 0\right)\right)\right)\right)
\end{aligned}
$$

For each path to a terminal 1, using the algebra of events gives

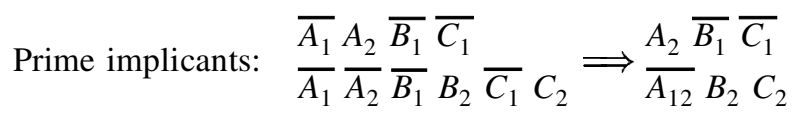

Nodes on a BDD path will represent failure or functioning of a particular component through different phases. These must be combined using the algebra of events given earlier, prior to evaluating the probability of the status required of that component.

Having considered each component encountered on a path, the probability of the path to a terminal 1 is evaluated as usual by taking the product of the probability of the component status. The phase failure is then obtained by summing the probability of each disjoint path

$$
Q_{2}=q_{A_{2}}\left(1-q_{B_{1}}\right)\left(1-q_{C_{1}}\right)+\left(1-q_{A_{1}}-q_{A_{2}}\right) q_{B_{2}} q_{C_{2}}
$$

Phase 3

The BDD representation for the fault tree representing failure during phase 3 is

Prime implicants:

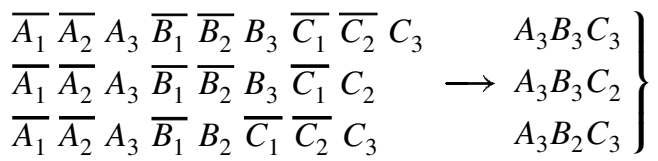

$$
\begin{aligned}
& \longrightarrow A_{3} B_{3} C_{23} \\
& Q_{3}=q_{A_{3}} q_{B_{3}} q_{C_{23}}+q_{A_{3}} q_{B_{23}} q_{C_{3}}-q_{A_{3}} q_{B_{3}} q_{C_{3}}
\end{aligned}
$$

Therefore, it can be seen that the unreliability of each of the phases as found by the BDD method is identical to that obtained using fault tree analysis [equation (3)].

\section{CONCLUSIONS}

1. The accurate assessment of mission unreliability for systems with non-repairable components operating over 
a sequence of phases can be performed using noncoherent fault tree structures.

2. The direct quantification of the fault trees is frequently problematic for even moderately sized problems owing to the size and complexity of the resulting logic functions.

3. Fault tree modularization methods provide some reduction in the size of the problem but not enough for this alone to offer a practical solution method.

4. The use of binary decision diagrams (enhanced to account for the phased nature of component failures) to calculate the failure probability of each phase in the mission provides an efficient and accurate means of evaluating the mission reliability.

\section{ACKNOWLEDGEMENTS}

The work described in this paper was conducted as part of a research project funded by the Ministry of Defence. The views expressed are those of the authors and should not be considered as those of the Ministry of Defence. The authors would like to thank Richard Denning, MOD, for his input to the research described in the paper.

\section{REFERENCES}

1 Esary, J.D. and Ziehms, H. Reliability of phased missions. Reliability and Fault-Tree Analysis, 1975, pp. 213-236 (Society for Industrial Applied Mathematics, Philadelphia, Pennsylvania).

2 Burdick, G.R., Fussell, J.B., Rasmuson, D.M. and Wilson, J.R. Phased mission analysis: a review of new developments and an application. IEEE Trans. Reliability, April 1977, R-26, 43-49.

3 Clarotti, C.A., Contini, S. and Somma, R. Repairable multiphase systems-markov and fault-tree approaches for reliability evaluation. In Synthesis and Analysis Methods for Safety and Reliability Studies (Eds G. Apostolakis, S. Garribba and G. Volta), 1980, pp. 45-58 (Plenum Press, New York).

4 Smotherman, M. and Zemoudeh, K. A non-homogeneous Markov model for phased mission reliability analysis. IEEE Trans. Reliability, 1989 December, 38, 585-590.

5 Smotherman, M. and Geist, R.M. Phased effectivenessusing a nonhomogeneous Markov reward model. Reliability Engng. and Syst. Safety, 1990, 27, 241-255.

6 Reay, K. and Andrews, J.D. A fault tree analysis strategy using binary decision diagrams. Reliability Engng. and Syst. Safety, 2002, 78, 45-56.

7 Sinnamon, R.M. and Andrews, J.D. Improved efficiency in qualitative fault tree analysis. Qual. and Reliability Engng. Int. 1997, 13(5), 293-298.

8 Sinnamon, R.M. and Andrews, J.D. Improved accuracy in qualitative fault tree analysis. Qual. and Reliability Engng Int. 1997, 13(5), 285-292. 